\title{
LOS USOS RECREATIVOS Y TURÍSTICOS DE LOS ESPACIOS NATURALES PROTEGIDOS. EL ALCANCE DEL OCIO EN EL MEDIO NATURAL DE MALLORCA ${ }^{1}$
}

\author{
Macià Blázquez Salom²
}

\section{RESUMEN}

El propósito de este trabajo ha consistido en la realización de un estudio exploratoriodescriptivo, mediante métodos inductivos basados sobre todo en el trabajo de campo, de la distribución geográfica de las actividades recreativas y turísticas en el medio natural de Mallorca. Complementariamente, se han identificado y evaluado los impactos ambientales y los conflictos de uso generados por dicha relación, para finalmente realizar propuestas de planificación y gestión de las actividades de ocio en el medio natural, mediante el análisis de experiencias de planificación y gestión del medio natural, principalmente en el continente europeo.

La diagnosis desarrollada identifica, caracteriza y categoriza las áreas de uso recreativo intensivo en el medio natural (AURIMN): primero, de uso más masivo, mayoritariamente bañista con instalaciones; segundo, las áreas de picnic invernal oficiales con instalaciones; tercero, las áreas de tránsito motorizado, ciclista y hípico; y finalmente, los itinerarios excursionistas más referenciados en las guías de excursionismo.

Palabras clave: recreación, turismo, espacios naturales protegidos y Mallorca.

\begin{abstract}
The main aim of our PhD thesis has been to develop an exploratory and descriptive study of the geographical distribution of outdoor and tourist activities in the natural areas of Majorca, by mean of inductive methods and based mainly on surveying. Complementary, the environmental impacts and the use conflicts caused by those uses have been identified and evaluated. Finally, proposals of planning and management of outdoor activities have been developed, through the analysis of planning and management experiences, mainly within the European continent.
\end{abstract}

1 El trabajo que se presenta es un resumen de nuestra tesis doctoral titulada «Els usos recreatius i turístics dels espais naturals protegits. L'abast del lleure al medi natural de Mallorca», dirigida por el Dr. Onofre Rullan, que ha sido leida y evaluada con el apto cum laude (15-11-96).

2 Departament de Ciències de la Terra, Universitat de les Illes Balears. Carretera de Valldemossa, km. 7,5, 07071 PALMA. Tel. (9) 71 173164, fax (9) 71173184 y e-mail: dctmbs4@ps.uib.es. 
Our diagnosis identify, characterise and categorise the intensive outdoor recreation areas in the designated sites of Majorca: first, by their massive bathing use with installations' presence; second, by their winter picnic use, in areas with public equipment; third, by their motorised, cycling and horse-riding use; and finally, by the frequency in which pedestrian paths are described in hiking guides.

Key words: outdoor recreation, tourism, designated natural areas and Majorca.

\section{Introducción, contexto geográfico y conveniencia del estudio}

Mallorca tiene ya una larga historia como destino turístico de masas. En la actualidad, visitan la isla al cabo del año, tantos turistas como diez veces su población residente (6.159.249 turistas respecto a 629.445 residentes en 1995). Este fenómeno social ha provocado una intensificación de uso territorial que se manifiesta en: la extensión de la urbanización del litoral así como de edificaciones en el medio rural, una densa red viaria que promueve la primacía del automóvil, y en general el aumento del consumo de recursos, sobre todo energía y agua, así como del incremento de la generación de residuos.

La primera respuesta social a esta degradación ambiental fue en defensa de los espacios naturales. Esta presión inicialmente ecologista congregó, a medio plazo, los intereses del empresariado turístico, alarmado por la imagen comercial negativa del cuño «balearización».

La aprobación de la Ley 1/1991 de Espacios Naturales (LEN), que protege urbanísticamente el $38 \%$ del territorio mallorquín, ha sido considerada como la culminación de esta campaña conservacionista, pese a que excluye algunas áreas y no erradica la rurubanización (BLÁZQUEZ, 1992).

Paralalelamente, los espacios naturales protegidos (en adelante ENP) se han popularizado y su uso recreativo y turístico se ha masificado. La problemática que ello conlleva, de aparición de conflictos de uso e impactos ambientales, se agrava por el hecho que la protección otorgada por la LEN es únicamente urbanística; es decir, no comporta gestión alguna del patrimonio y recurso natural.

Tan sólo el 4,11\% del territorio mallorquín, 3 parques naturales y 1 parque nacional, dispone de figuras de gestión del medio natural para la conservación de sus valores ecológicos y la gestión de su uso público.

\section{Objetivos del estudio}

El objetivo básico de nuestra investigación ha sido la determinación del alcance de los usos recreativos y turísticos en el medio natural de Mallorca, haciendo especial hincapié en su afección a los espacios naturales protegidos urbanísticamente, pero no gestionados.

Dentro de este propósito, ha jugado un papel preeminente la caracterización y categorización de las áreas de uso recreativo intensivo en el medio natural (en adelante AURIMN), especialmente por lo que respecta a la tipología e intensidad de sus usos y a sus características geográficas. Asimismo, se han identificado y evaluado los impactos ambientales y los conflictos de uso en dichas áreas.

Finalmente, nos hemos planteado realizar propuestas de planificación y gestión sostenible de los ENP, con el objeto de optimizar su rendimiento recreativo y minimizar los procesos que dan lugar a su degradación como patrimonio y como capital natural. El análisis de las experiencias de gestión de ENP, en Mallorca y en todo el continente europeo, nos ha servido para definir los modelos de manejo factibles y recomendables. 


\section{Estudios precedentes}

Éste ha sido un proyecto pionero en las Islas Baleares. Algunos otros investigadores han realizado estudios semejantes, fuera de las Baleares, para una o más áreas en ámbitos regionales, con el objeto de definir el alcance de las actividades recreativas en el medio natural y sus impactos y conflictos ambientales y de uso. La gran mayoría de esos estudios culminan en propuestas de planificación y gestión de las problemáticas diagnosticadas.

Los que nos han servido de más útil referencia proceden del ámbito anglosajón. Rosemary Burton (1975) estudió el caso del área recreativa de Cannock Chase, en Staffordshire, mediante análisis geográfico, ecológico y psicológico, a través del cual detecta conductas gregarias que la inducen a prever una mayor facilidad de gestión de sus impactos ambientales. Susan A. Glyptis (1981a, 1981b y 1991) realizó un análisis semejante para dos espacios más de Inglaterra: Beverley Westwood y la Reserva Natural de Spurn. Ambas autoras defienden la utilización de la punta de uso como el mejor indicador ambiental y psicológico: «Reflejar en un mapa al público visitante como agente de daño requiere idealmente un inventario completo e instantáneo en la hora punta.» (BURTON, 1975, pp. 154). Glyptis destaca, a su vez, las ventajas del método de observación y recuento instantáneo: provee de información precisa sobre la distribución de visitantes, tanto en el espacio como en el tiempo, puede servir para estimar la duración media de las visitas y permite establecer la estabilidad del uso, mediante la realización de diversas muestras.

Los equipos de investigación de los Servicios Forestales del Departamento de Agricultura de los Estados Unidos de América llevan a término estudios sobre la afluencia de visitantes al medio natural (CORDELL et alii, 1990, ENGLISH et alii, 1993) y sus implicaciones ambientales (COLE, 1982; COLE, 1985 y COLE, 1989), socioeconómicas (CORDELL et alii, 1993) y psicológicas (STANKEY, 1980). Sus publicaciones tienen como finalidad su uso por parte de gestores y vigilantes de áreas recreativas de uso intensivo en el medio natural.

Por lo que respecta al papel social y territorial de los ENP, Xavier Campillo (1992 y 1994) nos proporciona un detallado análisis del conflicto entre conservación de la naturaleza y promoción socioeconómica en Baden-Württemberg. Su tocayo, compatriota y contrincante argumental en dicha polémica es Xavier Carceller (1986) que defiende el papel de promoción socioeconómica de los espacios naturales.

Algunos naturalistas, como es el caso de Adam Watson (1991; LANCE, THAXTON y WATSON, 1991), que estudia problemáticas ambientales debidas a la sobrefrecuentación en los Cairngorms, en las montañas Grampianas escocesas, destacan la falta de datos fiables sobre la extensión de la presión recreativa en espacios naturales, que se puedan relacionar con sus efectos y su gestión efectiva.

El equipo de ecólogos del Centro de Investigación de Espacios Naturales Protegidos Fernando González Bernáldez (GÓMEZ-LIMÓN, 1992; DE LUCIO et alii, 1992; GÓMEZ-LIMÓN et alii, 1993; GÓMEZ-LIMÓN et alii, 1994 y GÓMEZ-LIMÓN y DE LUCIO, 1994) han desarrollado sus estudios en torno a la problemática generada por la sobrefrecuentación en el Parque Natural de la Cuenca Alta del Manzanares, Madrid. Los estimadores de impacto que establecen en sus estudios (GÓMEZ-LIMÓN y DE LUCIO, 1993 y 1994) son los que hemos adoptado para nuestras muestras: acumulación de residuos, suelo desprovisto de vegetación y exhumación de raíces. Sus estudios también atañen a las motivaciones de los visitantes del Parque (DE LUCIO y MÚGICA, 1994; MÚGICA, 1994; GÓMEZ-LIMÓN et alii, 1994), dando como resultado la atracción del arbolado, la presencia de agua y la accesibilidad. La proyección propositiva no está ausente de sus estudios, basándose en tres conceptos principales: educación, persuasión y disuasión. Un 
estudio concreto, particularmente semejante al que hemos realizado en Mallorca, se centra en una muestra de las AURIMN de la Comunidad de Madrid (GÓMEZ-LIMÓN et alii, 1994); dicho estudio analiza la presencia de visitantes en las punta de uso en 31 de las 105 áreas detectadas. Sus muestreos abarcaron, únicamente, los fines de semana de la primavera y el verano de 1993, para los cuales dicho equipo contó con la participación de los agentes forestales de la Agencia de Medio Ambiente de la Comunidad de Madrid. Sus resultados destacan el predominio de actividades sedentarias (60\% de los visitantes) y su concentración temporal en la punta de uso (80 \%).

Otro ejemplo de análisis de preferencias lo constituye la tesis doctoral de Mercedes de Frutos (1993), que estudia una muestra de 13 áreas recreativas de la Comunidad de Madrid, de entre 119 AURIMN identificadas. En su estudio extrapola los resultados de la muestra a la totalidad de casos para definir el alcance del recreo en Madrid.

\section{Métodos de análisis}

El estudio de las relaciones entre el hombre y su entorno deben fundarse en el análisis geográfico exploratorio. Sus objetivos deben ser descriptivos, causales, explicativos, predictivos y propositivos. Tal y como ya se ha anunciado, aunque nuestro estudio abarca todo el espectro del análisis exploratorio, su método es esencialmente descriptivo-explicativo y de diagnóstico de esta función del medio natural.

\subsection{Delimitación del ámbito de estudio}

Ante tal presupuesto, nuestro estudio realiza, en primer lugar, el análisis del medio natural de Mallorca, definido por los catálogos de espacios naturales (DDAA, 1992a; DDAA, 1992b; COPOT, 1987 y INESE, 1985) que culminaron en la promulgación de la Ley 1/91 de Espacios Naturales (véase mapa ${ }^{\circ} 1$ ).

Asimismo, se estudian otras figuras de protección y gestión del medio natural aplicadas en Mallorca, tales como la de paraje pintoresco de la Serra de Tramuntana y los montes de utilidad pública.

Con tales apartados se delimita el ámbito territorial de nuestro estudio, los espacios naturales protegidos de Mallorca, en tanto que estos presentan las situaciones de mayor tensión entre la conservación del medio natural y su uso turístico y recreativo. En total, la Ley 1/91 individualiza 47 ENP en Mallorca, a los que añadimos las islas adyacentes de sa Dragonera y Cabrera, así como las comunas municipales, no protegidas por la LEN pero de propiedad y vocación pública, del Puig de Santuïri y la Comuna de Llorito.

El reconocimiento territorial directo realizado en nuestros estudios previos (DDAA, 1992a, BLÁZQUEZ, 1993, ESPACIOS NATURALES, 1995; EQUIP TRAMUNTANA, 1988) y la consulta a personal de vigilancia, gestión y a otros investigadores nos ha permitido definir un total de 130 AURIMN, en base a su definición como áreas, en el medio natural, en las que se desarrollan actividades sedentarias o de tránsito, de manera recurrente y con cierta intensidad.

\subsection{Determinación del uso recreativo y turístico de las AURIMN}

Hemos escogido el establecimiento de la punta de uso — peaking — como la situación de mayor interés, por ser la de mayor estrés ecológico y de merma del rendimiento recreativo. Asimismo, es la aconsejada para el estudio de un cierto número de AURIMN 


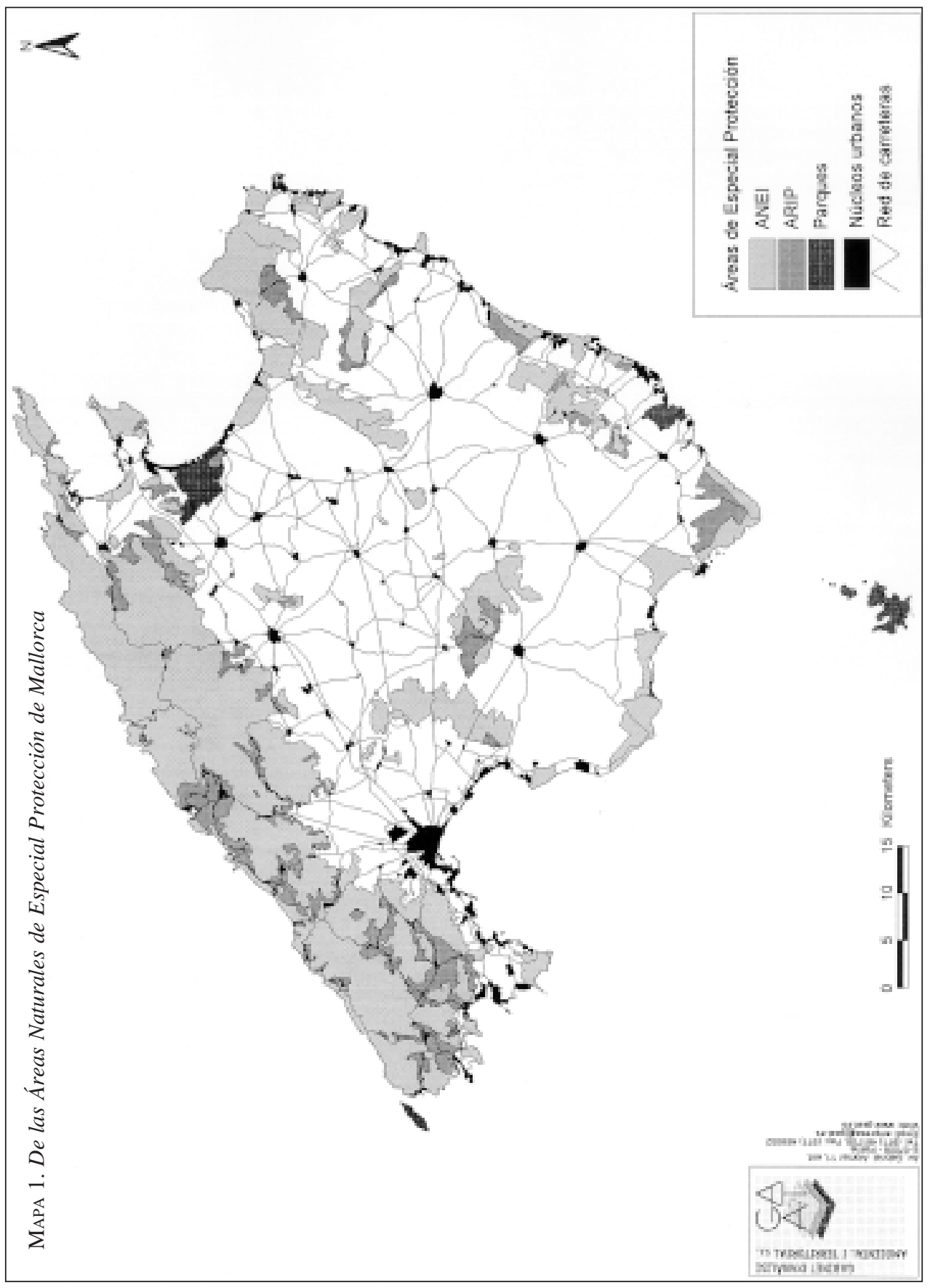


(BURTON, 1975; GÓMEZ-LIMÓN et alii, 1994; DE FRUTOS, 1993), tal y como es nuestro caso.

Nuestro proyecto de master (BLÁZQUEZ, 1993), en el cual estudiamos el comportamiento de la afluencia de visitantes a tres AURIMN escogidas, apriorísticamente, como de mayor disparidad y alto grado de conflicto, nos proporcionó la franja estacional y horaria dentro de la cual es esperable se alcance la punta de uso; esto es, entre las 12 y las $17 \mathrm{~h}$. Los métodos utilizados consistieron en el recuento a lo largo de transectos completos y consecutivos del área, junto con algunos aforos complementarios. También se ensayaron recuentos paralelos de individuos en el área y de vehículos estacionados en sus accesos.

Los recuentos realizados en las 120 AURIMN estudiadas en la tesis, tras la retirada de 10 de bajo uso, se hicieron coincidir con las puntas de uso esperables, en días laborables y en festivos, y a lo largo de las cuatro estaciones. Los recuentos incluían la clasificación de los individuos según las actividades que realizaban, de acuerdo con una ficha de campo que diferencia veintinueve tipos de actividad.

Se realizaron un total de 289 muestras, y por lo tanto, un promedio de 2,4 muestras por área.

La cifra correspondiente al número máximo de usuarios registrados para cada uso, de cada unidad de análisis, se ha utilizado para definir el índice de especialización funcional de cada AURIMN respecto de las demás, mediante la aplicación del índice de Nelson; el cual consiste en el cálculo de la media estadística de toda la serie y de su desviación estándar, para luego determinar grados (en nuestro caso hemos escogido hacer 5) de especialización funcional para cada uso, a partir del establecimiento de intervalos.

De otro lado, entrevistamos a todos los guardas forestales de la isla, así como a otro personal de vigilancia y gestión de las AURIMN, los cuales nos proporcionaron una visión más completa y extensa en el tiempo, para acabar de definir cualitativamente los usos de las AURIMN y sus intensidades, así como para afinar los momentos escogidos para los muestreos, a lo largo de las campañas de campo.

En un caso concreto, particularmente paradigmático, la playa de es Trenc, realizamos un total de 173 encuestas, a residentes y turistas, con el objeto de determinar su grado de satisfacción y su percepción de la saturación que llega a padecer dicha playa (BLÁZQUEZ, 1995). En este sentido, nuestro objetivo fue correlacionar la saturación en la punta de uso con una pérdida de rendimiento recreativo del área.

\subsection{Determinación de las características geográficas de las AURIMN}

Nuestro objetivo final, de hacer útil la determinación del alcance de las actividades recreativas en el medio natural, precisaba de la calificación de las áreas de estudio, para después correlacionar tipos y niveles de uso con las cualidades de las áreas.

Así pues, se definieron para cada AURIMN: su dimensión, su nivel de artificialidadnaturalidad según el tipo de ocupación del suelo, la presencia de cobertura arbórea, su litoralidad o vecindad a la mar, su distancia a Palma por carretera y la presencia de: aparcamiento delimitado para más de diez coches, bar, alquiler de tumbonas y sombrillas, venta de leña, instalaciones de picnic y váters.

Los impactos ambientales identificados en las AURIMN a resultas de las actividades estudiadas se cuantificaron, en función de los de mayor significación según estudios realizados en el Parque Regional de la Cuenca Alta del Manzanares (GÓMEZ-LIMÓN et alii, 1993 y GÓMEZ-LIMÓN y DE LUCIO, 1994): acumulación de desechos, denudación del suelo y descalzamiento de raíces de árboles. 
Los grados de especialización funcional de las AURIMN (79, después de una criba de las menos importantes, es decir, entre otras, las que no dan índices de Nelson igual a superior a uno para ningún uso) más las variables que las caracterizan geográficamente constituyen una matriz de información espacial (MIE), de treinta variables en total, a la cual hemos aplicado el método de análisis factorial de tipo exploratorio. Éste es un método inductivo orientado a desvelar las dimensiones subyacentes de las variables caracterizadoras de las unidades de análisis, con el objeto de determinar las concomitancias de los rasgos que las caracterizan.

En una primera aplicación de este método se nos definen ocho factores, de los cuales los tres primeros concentran el 51,2 \% de la varianza. La posterior reducción de la MIE, para un segundo proceso de análisis factorial, escogiendo las 13 variables más explicativas del primer proceso, resulta en el mantenimiento de los mismos tres factores iniciales ${ }^{3}$.

\subsection{El análisis de las guías de excursiones para inferir la presión excursionista}

Ciertos usos itinerantes, como es el caso del excursionismo, no se ciñen a la frecuentación de AURIMN. Con el objeto de incorporar este caso a nuestro estudio, hemos cuantificado la frecuencia con que se describen las excursiones a pie por la isla de Mallorca en un total de 38 publicaciones (libros, folletos y mapas; en catalán, castellano, inglés, alemán y algún otro idioma), transformando su incidencia territorial al número de veces que dichas excursiones atraviesan cada una de las cuadrículas quilométricas de la malla definida por la proyección UTM. Esta puntuación se ha ponderado según la importancia de la guías, asignándoles pesos en función de: su número de ediciones, su antigüedad, su tirada, las lenguas en que se han publicado, su precio o gratuidad, su difusión y la claridad expositiva de sus itinerarios. Finalmente se ha utilizado un programa cartográfico de interpolación para reflejar el número de referencias en las guías de excursión ponderadas, como una medida de la presión excursionista inducida en el medio natural de Mallorca, Cabrera y sa Dragonera.

Este método se ha ratificado mediante la inclusión de las tres excursiones que nos aparecieron como las más citadas en un estudio previo (BLÁZQUEZ, en prensa) en el análisis de especialización funcional, el cual les otorga índices apreciables.

La interpretación de dichos mapas, en los que además diferenciamos las guías de uso mayoritario de residentes de las de uso turístico, nos permite diferenciar las rutas preferidas.

\subsection{El análisis del rendimiento de las áreas recreativas o de picnic oficiales}

Además de incluir las áreas con instalaciones de picnic de la administración pública (del Servicio de Conservación de la Naturaleza, SECONA, del Gobierno Balear) en el análisis factorial de las AURIMN, hemos realizado un análisis de su rendimiento mediante la aplicación de los índices de densidad de usuarios por hectárea del National Playing

3 El acierto del uso de los índices de Nelson en la MIE, en lugar de los máximos de usuarios por usos, se constató mediante la realización de un análisis factorial con estos otros datos, que resultó en la definición de los mismos tres primeros factores, pero con la desvinculación de la presencia de instalaciones y el uso masivo de picnic; con lo cual se nos confirma la grave ineficiencia de la actual red de áreas oficiales, dado que las AURIMN más caracterizadas por la masividad de este uso no coinciden con las que poseen instalaciones oficiales. 
Fields Association (GB) y del National Recreation and Sport Association (EUA) (DÍAZ SEGOVIA, 1991).

\subsection{El uso de las ermitas en romerías y «pancaritats»}

Estos usos tradicionales del campo mallorquín han sido estudiados mediante la realización de recuentos, durante la Semana Santa de 1995 en 12 del total de 35 ermitas que son utilizadas en estos actos, junto con el uso de información oral, noticias de prensa y de consulta bibliográfica (JANER, 1992). El método de muestreo utilizado ha sido idéntico al usado en las AURIMN. Pese a todo, su uso esporádico no las hace equiparables al uso cotidiano, al menos estacionalmente, que caracteriza a las AURIMN.

\subsection{El análisis de la frecuentación de los Parques}

Las estadísticas de afluencia de visitantes a los cuatro parques del ámbito de estudio (Parque Nacional del archipiélago de Cabrera y Parques Naturales de s'Albufera, Mondragó y sa Dragonera), junto con los datos de otros parques del continente europeo, nos sirven para establecer los términos de referencia comparativa, así como para analizar los métodos y técnicas ensayadas y acreditadas de gestión de las actividades recreativas y turísticas en espacios naturales.

Se han recogido sus datos de puntas de uso, equiparables a los caracterizadores de las AURIMN, así como de su número total de visitantes anuales.

Los máximos puntuales de afluencia han sido tratados, con propósito comparativo, junto al conjunto de datos de las AURIMN, con la aplicación del índice de Nelson.

\subsection{Identificación de impactos ambientales y conflictos de uso}

La identificación de impactos ambientales se basa en la metodología expresada en el apartado 4.3, mientras que la identificación y evaluación de los conflictos de uso se realiza mediante su atención en las visitas de campo, las encuestas a usuarios y las entrevistas al personal de vigilancia y gestión, de entre el que destaca la totalidad de los guardas forestales de la isla (17 con responsabilidad sobre el terreno, dos jefes de área y un superintendente).

\subsection{Propuestas de planificación y gestión de ENP de uso recreativo y turístico}

Nuestro análisis parte tanto del análisis de la bibliografía sobre gestión sostenible del uso turístico de ENP (SCHOFIELD, 1995; HEUKEMES, 1993; JUBENVILLE y TWIGHT, 1993; ROGGERBUCK, WILLIAMS y WATSON, 1993; MARIN CABRERA, 1991; KUSS, GRAEFE y WASKE, 1990; MARTIN y UYSAL, 1990; BAIRD y IVE, 1989; NATURE CONSERVANCY COUNCIL, 1988; HAMMITT y COLE, 1987; KNUDSON, 1984; MACEWEN, 1982; MILES y SEABROOKE, 1977, entre otros), como de nuestra experiencia profesional de colaboración con gestores de ENP del continente europeo, como encargado del Secretariado Mediterráneo de EUROSITE, que es una organización no gubernamental que tiene por objeto la mejora en las técnicas de gestión de estas áreas, para la cual coordinamos la realización de manuales al respecto (EUROSITE, 1994 y 1996). 


\section{Resultados}

\subsection{Determinación del alcance de la recreación en el medio natural de Mallorca}

Éste ha sido el objetivo, y consecuentemente, el resultado principal de nuestro estudio, el cual se basa en la categorización de las AURIMN, la identificación de las rutas excursionistas más populares, el análisis del rendimiento de las áreas oficiales de picnic, el uso recreativo de las ermitas y la afluencia de visitantes a los parques.

\subsubsection{Caracterización y categorización de las AURIMN}

La presencia de instalaciones es la característica geográfica más relevante de las AURIMN, en tanto que atraen y concentran sus usos. Por lo que respecta a las tipologías paisagísticas cabe destacar la diferenciación de cuatro ámbitos: primero las calas y arenales, en los que coadyuva la presencia de arbolado; segundo, los bosques; tercero, las cimas y miradores preeminentes; y finalmente, los tramos de litoral no urbanizados con fácil acceso a la mar.

Las tipologías de usos que establecimos, a partir de estudios análogos y del trabajo de campo, fueron: el baño, el paseo, el ciclismo, la hípica, el picnic, la acampada, la acampada con caravanas, el tránsito motorizado, el fondeo y varada de embarcaciones y la pesca de caña en el litoral.

La tabulación y análisis estadístico, mediante el índice de Nelson, de los datos de frecuentación nos otorgan los siguientes resultados para cada uso:

Uso de baño: dos conjuntos de arenales exentos de urbanización dan los máximos de frecuentación. Destacan, con grado de especialización 5, las AURIMN de los arenales meridionales, es Trenc, con 3.600 usuarios y la playa nord-oriental de Cala Agulla con 2.710. Cabe añadir que ninguna cala se libra de números de bañistas relevantes.

Uso de paseo: las AURIMN que tienen un uso más intensivo de paseo contemplativo son monumentos naturales y miradores, que son destinos preferidos de excursiones colectivas, mayoritariamente en autocar. Cabe destacar los casos de la desembocadura del cañón cárstico del Torrent de Pareis, sa Calobra, con puntas de uso de hasta 1.925 usuarios (única con grado de especialización 5) y el Puig de Sant Salvador, en las sierras de levante, con hasta 632 usuarios y grado 4 . Los tres itinerarios de excursiones a pie muestreados dan índices apreciables (índice Nelson 1) para este uso.

Uso de ciclismo de todo terreno: los dos espacios de uso más intensivo son periurbanos y litorales: el cabo oriental denominado Punta de n'Amer y el intersticio litoral periurbano de Palma, es Carnatge, ambos únicos de grado 5.

Uso hípico: los valores más elevados se nos presentan en áreas del entorno de núcleos turísticos intensivos, así como en un caserío de montaña, en el cual se organizan excursiones turísticas.

Uso de picnic: en primer lugar nos aparecen áreas de picnic oficiales, de montaña y de interior; las áreas de mayor intensidad de uso son (véase mapa $n^{\circ} 2$ ): el puig de Santa Magdalena, en los contrafuertes orientales de la sierra de Tramuntana, con hasta 540 usuarios (grado 5), Manut con hasta 377 usuarios (grado 4) y Ca s'Amitger con hasta 278 usuarios (grado 3) ambos en el entorno del monasterio de Lluc. Y de otro lado, con uso tan o más masivo, nos aparecen áreas costeras y sin instalaciones oficiales, es decir «espontáneas», que ocupan intersticios marginales, a menudo de suelo urbanizable o «reserva urbana»; las áreas de uso máximo son: el entorno del hotel Delta, situado junto al cabo que cierra a levante la bahía de Palma, con hasta 666 usuarios (grado 5), Cala Agulla con hasta 


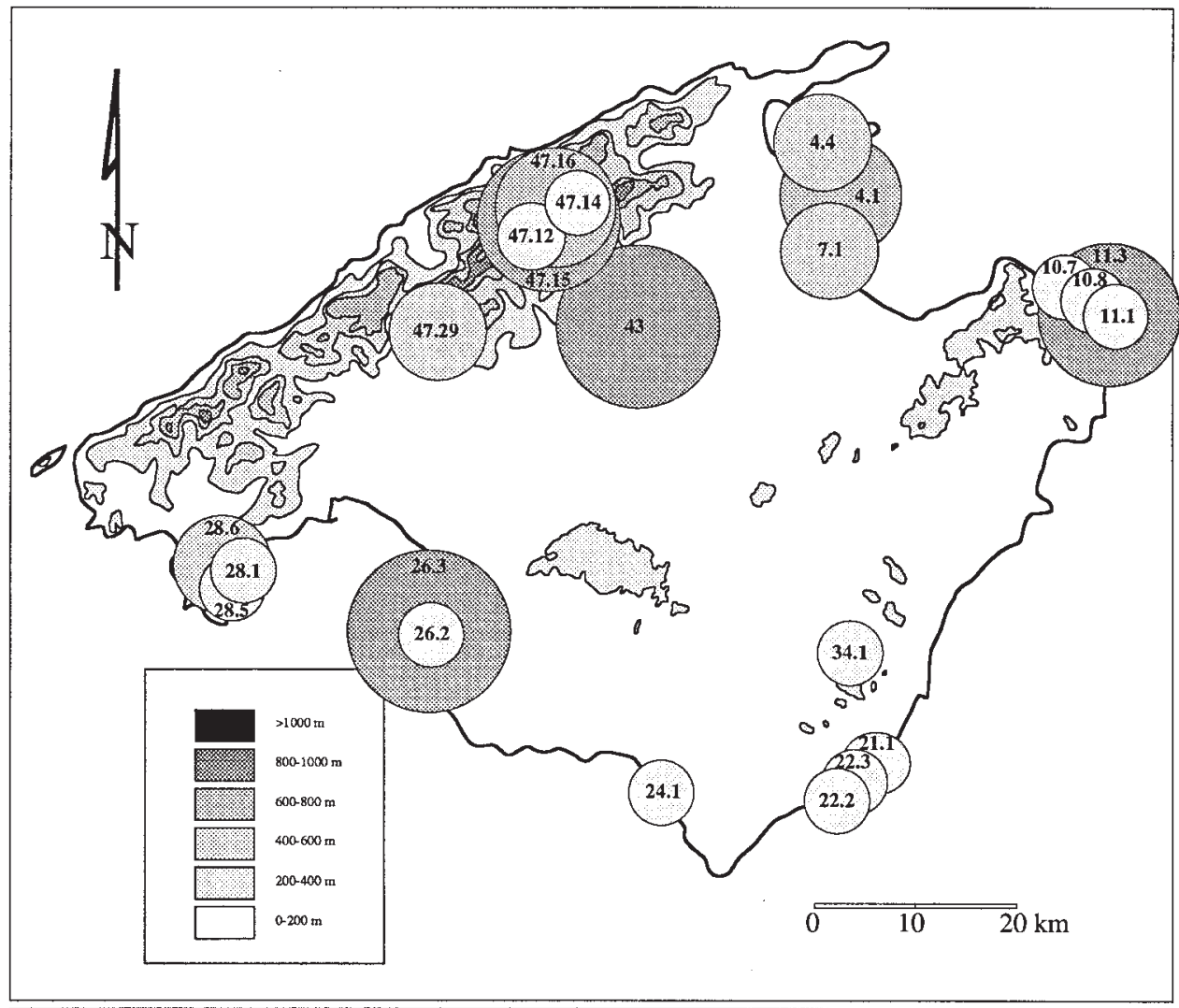

\section{Áreas de Uso Recreativo Intensivo en el Medio Natural de Mallorca de grado de especialización significativo}

4.1.- Alcanada - es Pi Tort

4.4.- S'Illot

4.5.- S'Ermita

7.1.- Es Comú

10.7.- Cala Mitjana

10.8. - Cala Torta

11.1.- Plata de sa Mesquida

11.3.- Cala Agulla

22.1.- Caló des Burgit

22.2. Fonts de n'Alis

22.3.- S'Amarador

24.1.- Platja de s'Estanyol - ses Arenes - es Coto
26.2.- Davallador de ses Olles

26.3.*- Hotel Delta- es Puigderrós

28.1.- Cala Falcó

28.5.- Portals Vells

28.6*.- A.R. de Son Ferrer

43.- $\quad$ Puig de Santa Magdalena

47.12. - A.R. de la Cometa Negra

47.14.- A.R. des Pixarells

47.15. - A.R. de Manut

47.16.- A.R. de Ca s'Amitger

47.29.- Sa Comuna de Bunyola

Grados de especialización funcional
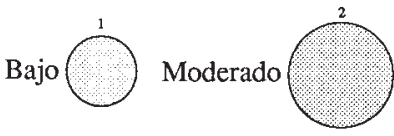

Elevado

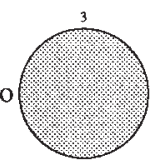

Alto

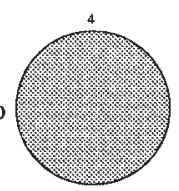

Máximo

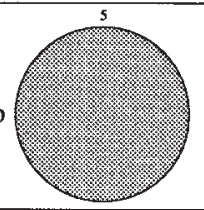

Fuente: elaboración propia

Mapa 2. De Áreas de uso Recreativo Intensivo en el Medio Natural de Mallorca. Mapa de especialización funcional. Tipología: uso de picnic. 
355 usuarios (grado 4) y Alcanada-es Pi Tort, cerrando la bahía de Alcúdia por el norte, con hasta 278 usuarios (grado 3). Sus usuarios son básicamente residentes.

Uso de acampada: el área de acampadas del monasterio de Lluc, que es semi-oficial, tiene el registro máximo (648 usuarios, única de grado 5), mientras el resto de áreas tienen concentraciones bajas y son del todo «espontáneas» e ilegales.

Uso de acampada con caravanas: también se repite la «espontaneidad» alegal junto a la costa, con máximos en Cala Agulla, con 52 usuarios que le otorgan grado 5, y hotel Delta, 20 usuarios y grado 4, y en la sierra, con el máximo en Lluc, con 32 usuarios y grado 5.

Tránsito motorizado: las áreas de mayor uso se asocian con litoralidad, paseo y acceso al mar. Los máximos se alcanzan en: sa Punta de n'Amer con una punta de uso de 162 usuarios y grado 5, s'Algar, junto al puerto de Felanitx, con hasta 88 usuarios y grado también 5, y Cala Falcó, casi cerrando a poniente la bahía de Palma, con hasta 74 usuarios y grado 4 .

Fondeo y varada de embarcaciones: la concentración más elevada de áreas de fuerte uso se da en los arenales del sudeste, que también ostentan los máximos de usos de baño. Se trata de es Carbó, con 392 usuarios (grado 5), es Trenc, con 336 y la playa del Dolç, con 292 usuarios (ambos de grado 4). Esta coincidencia da lugar a graves conflictos de uso, debido al excesivo solapamiento de ambas actividades, a despecho de la distancia reglamentaria de seguridad.

Pesca costera de caña: se trata de usos no organizados, minoritarios y muy dispersos por todo el litoral. Pese a todo, se detectan concentraciones relevantes en cinco áreas.

El análisis factorial exploratorio aplicado al conjunto de datos geográficos y funcionales, que constituyen la MIE, permite identificar tres factores agrupadores de las AURIMN (véase mapa ${ }^{\circ} 3$ ):

En primer lugar las áreas de uso masivo, mayoritariamente de baño, con instalaciones. Este factor explica el $41 \%$ de la varianza. Las áreas de mayor puntuación son arenales muy publicitados como «playas paradisíacas»: tres áreas del entorno de es Trenc, con pesos factoriales de 3'8, 2'6 y 2; la playa de Cala Agulla, con un peso de 4'4 y en tercer lugar el grupo de calas del Parque Natural de Mondragó, escogido botón de muestra de las calas meridionales y orientales en Santanyí, con pesos entre 1'6 y 1'1.

Las áreas de uso de picnic invernal con instalaciones, es decir: mesas, bancos, fogones, venta de leña y aparcamiento. Este factor explica el $36 \%$ de la varianza. Tal y como comentábamos en el resumen de los resultados del análisis de especialización funcional, las áreas oficiales de picnic se localizan en fincas públicas, que son mayoritariamente de interior, dado su propósito inicial de reserva forestal. La mayor concentración de áreas de elevada puntuación se da entorno a Lluc, con cinco áreas recreativas de pesos entre 3’2 y 1'5, en bosques de encina, propios de la vegetación mesosupramediterránea. El monasterio, conocido como el corazón espiritual de Mallorca, ejerce un gran atractivo, en el corazón, también, de la montaña mallorquina por antonomasia: la sierra de Tramuntana. Otros pesos también elevados se alcanzan en ermitas del llano central de la isla, ubicadas sobre pequeños macizos de gran valor paisajístico y que también albergan encinares: el puig de Santa Magdalena (peso 3'6) y el puig de Sant Salvador (peso 2).

Las áreas de picnic litorales, alegales y de uso estival aún más masivo, no puntúan para este factor entre los pesos incluidos en el décimo decil ${ }^{4}$.

Las áreas con tránsito motorizado, ciclista y hípico. Este factor explica un $23 \%$ de la varianza. Un primer grupo de áreas de mayor peso consiste en destinos litorales rocosos y

$4 \mathrm{La}$ aplicación de percentiles a las puntuaciones factoriales ha proporcionado, en la tesis, un criterio discriminatorio para la representación cartográfica y la presentación de los resultados. 

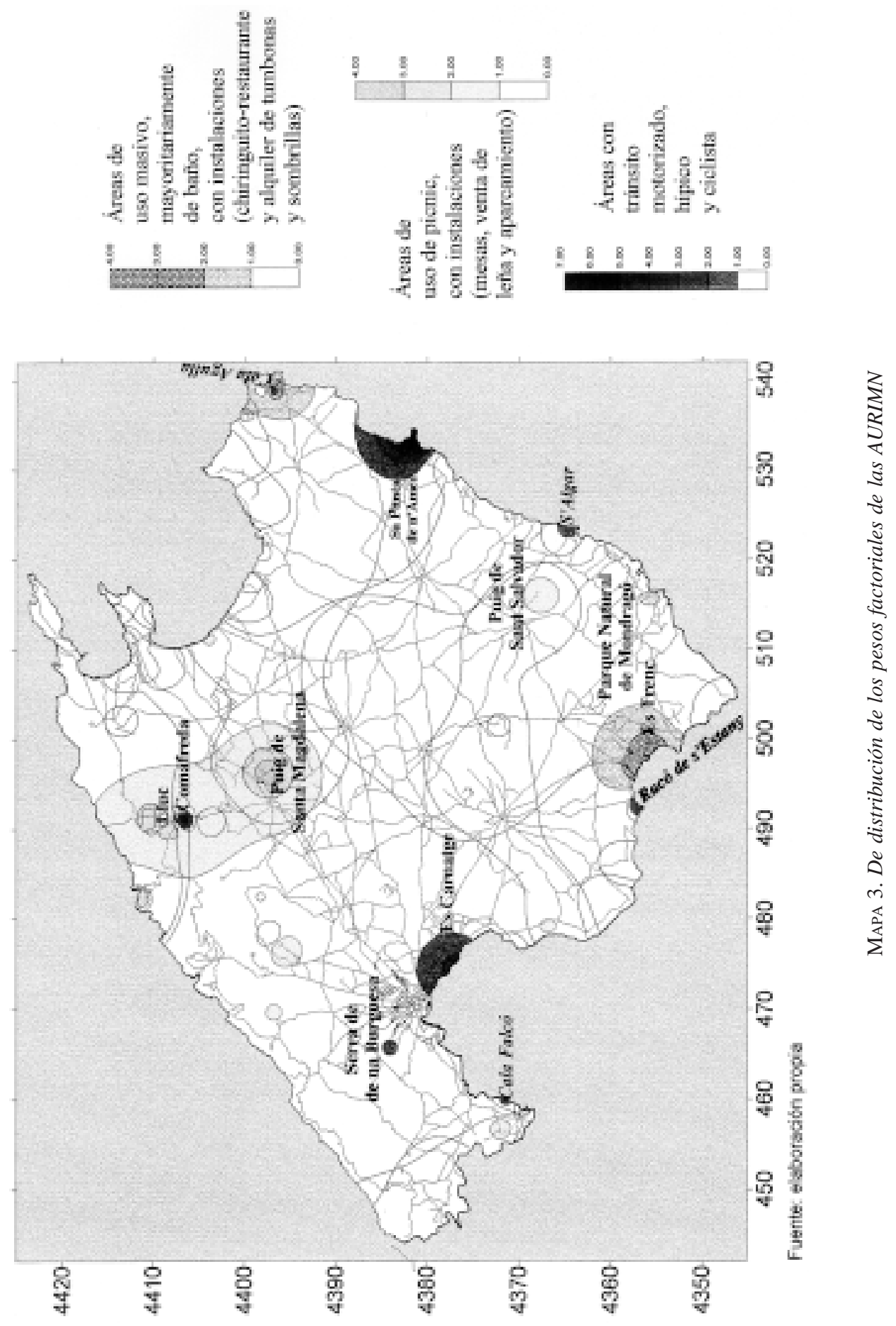

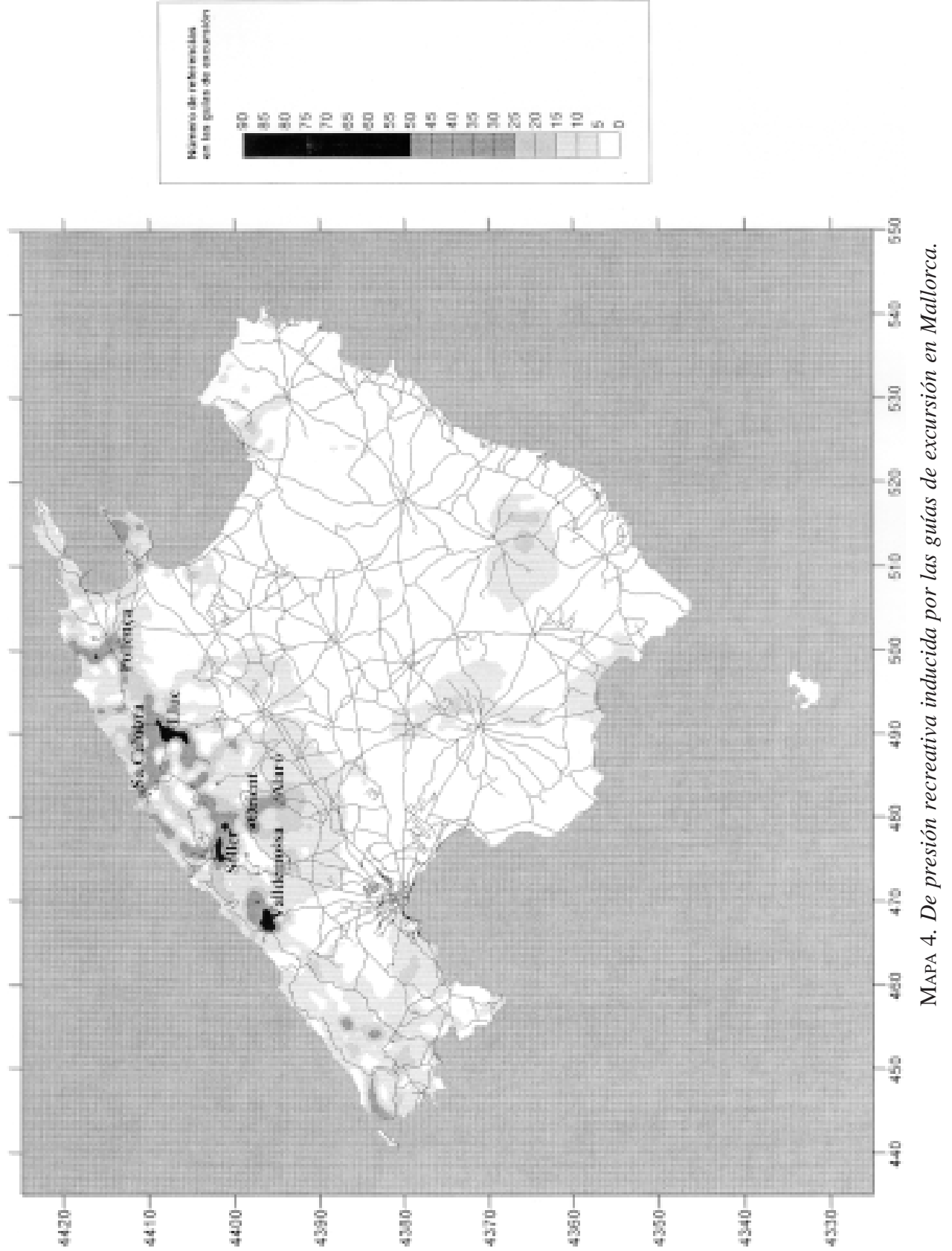
dunares, de carácter periurbano: la Punta de n'Amer, con la puntuación más elevada (6'1), es Carnatge (2’3), s'Algar (1'7), el Racó de s'Estany (1'4), Cala Agulla (1'3) y Cala Falcó (1’3). En segundo lugar, según este criterio de caracterización geográfica, se nos dan dos destinos de recreación de montaña: el camino del puig de Massanella (2'6), en el seno de un caserío, Comafreda, en el que se realizan excursiones organizadas en coches de todo terreno y la Serra de na Burguesa (1'1), que es el trasfondo montañoso de Palma.

Por otro lado, también podemos interpretar los resultados de la puntuación de las AURIMN para este último factor atendiendo a dos tipologías de áreas de uso recreativo de paseo rodado y a caballo: en primer lugar las de uso turístico y en segundo lugar las utilizadas mayoritariamente por residentes; con lo cual se apunta la segregación de usuarios.

\subsubsection{El análisis de la frecuentación excursionista inducida por las guías de excursión}

Los itinerarios más recomendados por las guías (véase mapa $\mathrm{n}^{\circ} 4$ ) recorren las sierras de la isla, especialmente la de Tramuntana. El mapa resultante muestra como el sector central de dicha sierra presenta un anillo de intenso uso excursionista que une Sóller, Lofra, Lluc, sa Calobra y sa Costera, dejando un vacío en su centro, que coincide con una zona más agreste, entre Moncaire, Monnàber, Son Torrella y los Binis. Dicho anillo se insiere entre los pueblos de Valldemossa, Alaró y Pollença, que son, junto con Lluc i Sóller, los principales centros excursionistas de Mallorca ${ }^{5}$.

Las tres excursiones escogidas para su análisis estadístico como AURIMN se encuentran entre las de mayor número de referencias por cuadrícula, entre 50 y 75 puntos: el Camí de s'Arxiduc a es Teix, al noroeste de Valldemossa; el Barranc de Biniaraix de ascenso a Lofra, marcando un extremo del anillo que une Sóller y Lluc; y el camino del puig de Massanella, que parte del monasterio de Lluc.

Dentro de este mismo intervalo de inducción a la frecuentación se identifican cuatro itinerarios más: el camino de ascenso al puig Tomir, el camino del Castell del Rei, sa Calobra en el extremo de las excursiones del Torrent de Pareis y de sa Costera, y Orient que es el vértice septentrional del arco que marcan las dos excursiones de ascenso al Castell d'Alaró.

Junto a la atracción que ejercen las sierras, también se aprecia una cierta atracción hacia el litoral, sobre todo por lo que respecta a los itinerarios preferidos por las guías dirigidas a excursionistas extranjeros.

Por último, cabe reseñar la potenciación del excursionismo naturalista, que auspician sobre todo guías institucionales, hacia las zonas húmedas de la isla: s'Albufera y es TrencSalobrar de Campos.

El análisis de los resultados correspondientes a las preferencias de las guías excursionistas para residentes (publicadas en catalán y/o difundidas a través de periódicos locales) decanta las áreas de mayor inducción hacia el entorno de Palma, las de mayor dificultad de acceso y alejándose de núcleos turísticos intensivos.

Por lo que respecta a las guías orientadas a excursionistas extranjeros, destaca la estrecha relación de muchas excursiones con los complejos turísticos litorales y en áreas de paisaje singular, diríamos incluso anecdótico, accesibles y menos atractivas para el excursionista residente. Este hecho evidencia la necesidad del negocio turístico de disponer de itinerarios cercanos y de libre paso.

5 Cabe señalar que pese a que las excursiones se dirigen al medio natural, los nucleos urbanos son puntos de paso obligados, por lo cual presentan los valores más elevados en nuestro análisis. 


\subsubsection{Análisis del rendimiento de las áreas de picnic oficiales}

Los conjuntos de mesas, bancos y fogones de torrada, instalados por la administración central (ICONA) y autonómica (SECONA) se ubican, casi exclusivamente, en fincas públicas seleccionadas con finalidad forestal, atendiendo al estereotipo cinematográfico y «continental» de búsqueda de frondosidad, sombra y entorno montañero.

La aplicación de los estándares de densidad de usuarios en instalaciones de picnic (que son de 40 a 100 usuarios por hectárea de capacidad máxima instantánea para picnic ligero) a las diez áreas muestreadas ha dado como resultado que siete lo excedan y que cuatro incluso exceden el estándar de picnic denso (200-300 usuarios/ha): Son Ferrer, Sant Salvador, Santa Magdalena y la Comuna de Bunyola.

Por otro lado, otra gran parte de las instalaciones tienen un uso escaso debido a su localización errónea, sin atender a las demandas reales y en función de la disponibilidad de suelo público.

Si a esta situación le añadimos la aparición de multitud de áreas de picnic de uso intensivo alegales y espontáneas (véase 5.1.1), se nos hace patente la desconexión entre la oferta pública y la demanda real. Las preferencias estivales de los usuarios demandan áreas equipadas en el litoral, en los espacios naturales intersticiales de los entornos residenciales y turísticos. Asimismo, cabría atender a la idoneidad de ubicar nuevas instalaciones en la periferia de Palma, con el objeto de interceptar a los usuarios que acuden a la llamada de la montaña los fines de semana; de manera que se redujese la necesidad de desplazamiento, se hiciese accesible este tipo de equipamiento mediante transporte público y se evitasen los impactos ambientales y conflictos de uso que se generan en las instalaciones situadas en el corazón de los espacios naturales, como es el caso del entorno de Lluc, la Comuna de Bunyola o Sant Salvador.

A nuestro entender, estas áreas cumplen una función social, territorial y ambiental muy importante. En primer lugar, el perfil socioeconómico proletario de sus usuarios denota el deseo de acercamiento al medio natural a través de una actividad de bajo coste, en la que la accesibilidad que proporciona el coche, y su propia presencia, son protagonistas. En segundo lugar, las áreas de picnic concentran un uso sedentario y intensivo, que de otra manera ocupa espacios no habilitados de manera espontánea, generando graves impactos ambientales y conflictos de uso. Por último, estas áreas son el primer estadio de acercamiento al medio natural y, por lo tanto, deben aprovecharse para educar y concienciar a la ciudadanía, propiciando un mayor conocimiento y respeto en la relación del hombre con la naturaleza.

\subsubsection{El uso de las ermitas en romerías y «Pancaritats»}

Las ermitas, santuarios, capillas y monasterios son instalaciones religiosas que se localizan mayoritariamente en entornos naturales. Sus usos tradicionales, de culto y peregrinación, se han extendido en la actualidad por los derroteros de la recreación y del turismo, desligados de su función tradicional y a menudo masivos.

Las ermitas, como denominación genérica, de uso más intensivo se han incluido en el análisis de AURIMN (es el caso de Cura, Santa Magdalena, Lluc, Sant Salvador, Bonany, etc.). Pero también hemos querido analizar la afluencia de usuarios que se da durante periodos festivos religiosos, en concreto las romerías de Semana Santa o «Pancaritats». Nuestros resultados muestran concentraciones máximas en: la ermita de Santa Magdalena, con hasta 1.604 usuarios, la ermita de la Mare de Déu de la Pau de Son Seguí, con hasta 842 usuarios la ermita de Bonany, con hasta 800 usuarios y el conjunto de el monasterio de 
Cura y del oratorio de Gràcia que, en un mismo día, concentran hasta 1.301 usuarios El castillo de Bellver, en el que se realiza el «Pancaritat» del «Dia de l'Àngel» de Palma, supera con mucho los datos recogidos en el resto de ermitas (no se dispone de datos oficiales o fiables y los reflejados por la prensa sitúan la afluencia entorno a 10.000), pero se trata de un parque urbano, que analizamos junto a otras figuras heterodoxas de Parques de Mallorca.

Aún sin desdeñar los problemas que surgen a raíz de esta masificación del uso, como es su enfrentamiento al modus vivendi pre-turístico y espiritual o la degradación del suelo y de la cubierta vegetal, valoramos positivamente el mantenimiento de una relación valorizadora de retales naturales en el entorno rural, como experiencia concienciadora del valor del patrimonio arquitectónico, cultural y natural.

\subsubsection{La frecuentación de los Parques}

Los Parques, como denominación genérica de los ENP gestionados mediante la aplicación de figuras de la Ley 4/89, suponen la aplicación de medidas de gestión efectiva, que es la herramienta fundamental para mejorar la calidad del uso público y para incrementar la capacidad de acogida de las AURIMN. Su tratamiento, a partir de las numerosas estadísticas oficiales de frecuentación, nos equipara los datos relativos a sus puntas de uso a la presencia de: entre 458 y 1.100 usuarios en el centro de interpretación de sa Roca, del Parque Natural de s'Albufera, situándolo como AURIMN de uso de paseo entre los índices Nelson 3 a 5; el puerto del Parque Nacional de Cabrera, con puntas de entre 271 y 652 usuarios, entre los índices 2 y 4; el puerto de es Lladó, único acceso del Parque Natural de sa Dragonera, con puntas de entre 187 y 448 usuarios, entre los índices 1 y 3; y, por último, el centro de información de ca na Martina, del Parque Natural de Mondragó, con 111 a 266 usuarios, entre los índices 1 y 2. Por lo tanto, sobre todo s'Albufera y Cabrera se equiparan a los índices alcanzados por los principales destinos de las excursiones colectivas en autocar, que tienen índices Nelson semejantes, de sa Calobra y de Sant Salvador.

El segundo análisis realizado, esta vez sobre el total de usuarios anuales y su densidad por hectárea, presenta la superación de la densidad media de diez parques europeos más los cuatro mallorquines (la selección de parques europeos se ha hecho sobre los presentados por HEUKEMES, 1993 como ejemplos de sobrefrecuentación), que es de 55 visitantes por hectárea y año, para los parques de s'Albufera, en un $67 \%$, y de sa Dragonera. Creemos que la diagnosis de saturación se extremaría aún más si considerásemos el área real en la que se concentra el uso de los Parques.

En términos generales, hemos constatado la falta de rigurosidad en la gestión de los Parques de Mondragó y de sa Dragonera, en los que no se realiza prácticamente ningún control de las tipologías y alcance de las actividades de ocio, así como de sus impactos ambientales y de conflictos de uso.

\subsection{Identificación de impactos ambientales y conflictos de uso}

Nuestra diagnosis es de sobreexplotación de los ENP, pero con malbaratamiento, es decir infrautilización, de sus valores patrimoniales reales. Son especialmente reseñables los impactos que degradan el patrimonio natural. Los impactos evaluados presentan las más altas correlaciones con los usos de picnic (correlación del 0,4$)$, de acampada $(0,4)$ y de acampada con caravanas $(0,3)$. Paralelamente, el cuarto factor del primer análisis factorial, que identifica las áreas con actividades de acampada, presenta el mayor índice de saturación de esta variable $(0,4)$. 
Por otro lado, la pérdida de rendimiento turístico debida al abarrotamiento y al desencanto se ha constatado en es Trenc, a partir de la encuesta a usuarios. Los resultados muestran que el $40 \%$ de los entrevistados destacan este hecho como principal queja respecto de las expectativas que se habían hecho de su visita a dicha playa. Pero, por otro lado, actividades como el picnic parecen valorar positivamente la aglomeración, en una muestra de actitud gregaria.

Otra fuente de conflictos de primer orden es la coexistencia de tránsito motorizado, identificado por nuestro tercer factor, y otras modalidades de paseo (como sucede en la Punta de n'Amer). También la coincidencia de actividades náuticas y baño provoca graves conflictos de uso, como en el caso paradigmático de las playas del entorno de es Trenc.

La insuficiencia e ineficacia de las áreas oficiales de picnic da lugar a la aparición de numerosas áreas de picnic espontáneas, sobretodo, en competencia por los intersticios costeros con otros usos. Especialmente, la residencialización, con su componente de cercado de fincas y accesos, desplaza este uso, vinculado a recreacionistas de inferior poder adquisitivo.

El excursionismo ha aprovechado, de antaño, el respeto consuetudinario a caminos y cañadas tradicionales, así como el talante generoso y hospitalario del payes mallorquín. Pero en la actualidad y de cada día más, la propiedad rural cambia de manos. La procedencia urbana de los «neorurales» y la nueva función residencial, y no agraria, a la que destinan sus propiedades genera graves disputas con los excursionistas en cuanto al derecho público de paso. Entrevistas y misivas de autores extranjeros de guías de excursionismo nos atestíguan que el conflicto alcanza ya a turistas extranjeros que planean sus vacaciones de treking en Mallorca para luego encontrar las barreras cerradas a cal y canto.

En términos más generales, el uso turístico y recreativo de ENP debe fundarse en sus valores patrimoniales. Pero se nos hace evidente que la explotación actual de estos recursos es trivial y, diríamos incluso, «tropicalizada». En lugar de orientar e informar a los turistas sobre los verdaderos valores naturales o culturales, se disfrazan las AURIMN conforme a los tópicos y estereotipos cinematográficos, más bien tropicales. A esta consecuencia de la falta de institucionalización de la conservación y el uso del patrimonio se une una gravísima situación de inseguridad para los usuarios, debida a la falta de planes de evacuación de las AURIMN, así como de otras medidas de protección, vigilancia y auxilio. Los casos más fragantes de infrautilización los encontramos en los ENP periurbanos intersticiales, que se caracterizan por padecer procesos de degradación acelerados, con pérdida de valores patrimoniales y de capital natural.

\subsection{Propuestas de planificación y gestión de ENP de uso recreativo y turístico}

La medidas de gestión a aplicar para mitigar los conflictos de uso y los impactos ambientales de las actividades de ocio en el medio natural son:

* La planificación de estructuras de AURIMN diversificadas y jerarquizadas, en función de las demandas recreativas y sus capacidades de carga ecológica.

* La restauración y incluso recreación de ambientes atractivos para el ocio en el medio natural.

* La elaboración y aplicación de planes de uso y gestión que persigan la conservación del patrimonio y la racionalización de su uso.

* El establecimiento de límites de cambio aceptable en las AURIMN, al objeto de asegurar su rendimiento recreativo y la conservación de sus valores naturales.

* La aplicación de técnicas de gestión del ocio en el medio natural, tales como la 
zonificación, la temporalización, el racionamiento y la interpretación. Se priorizarán las herramientas de persuasión para canalizar y concentrar o dispersar los usos, según sus características y las cualidades ambientales del lugar.

La extensión de los propósitos de los actuales convenios forestales, entre la administración pública y los propietarios de fincas forestales para mejorar la producción silvícola o la prevención de incendios forestales, a la ejecución y financiación pública de dichas medidas de gestión del ocio se nos presenta como una posibilidad de rentabilización económica para las explotaciones agrarias que contienen AURIMN. Figuras semejantes se utilizan en Gran Bretaña con el nombre de acuerdos de gestión, management agreements, y de acuerdos de acceso, access agreements, junto con exenciones fiscales para hacer partícipes a los propietarios de ENP y AURIMN de sus funciones de conservación de recursos y uso público, siguiendo el principio de voluntariedad.

Por otro lado, la colaboración de organizaciones no gubernamentales conservacionistas está a la orden del día en el continente europeo, al objeto de rentabilizar el mantenimiento de niveles de uso público de ENP compatibles con su objetivo primordial de conservación del patrimonio. Tratándose de organizaciones no lucrativas, las aportaciones particulares y públicas adquiren matices filantrópicos, basados en el voluntarismo y en la subsidiariedad del estado del bienestar.

Como una primera aportación propositiva respecto al fenómeno analizado y frente a la falta de atención a las demandas recreativas en la selección y evaluación de ENP, hemos considerado adecuado concluir nuestro estudio con la definición de una nueva red diversificada y jerarquizada de ENP y AURIMN de Mallorca, en base a tres objetivos: librar de sobrepresión recreativa a las áreas más frágiles, satisfacer las demandas con las mínimas exigencias de desplazamiento y favorecer el acercamiento escalonado a la naturaleza, educando a los usuarios generalistas.

Sus principales elementos, estructurados según la escala geográfica a la que servirían, serían (véase el mapa $\mathrm{n}^{\circ}$ ):

\section{En el rango insular:}

I.1.La sierra de Tramuntana, bajo la tónica del reconocimiento del derecho de paso público y la necesidad de gestión efectiva, a través de medidas de vigilancia, señalización, interpretación y restauración. En este ámbito, la AURIMN de sa Calobra encabeza los destinos masivos de elevado interés naturalístico.

I.2.Las playas masificadas y multifuncionales: es Trenc y su entorno, Cala Agulla, es Comú de Muro y Mondragó; en las que la situación de degradación y conflicto de usos hace perentoria la aplicación de planes de gestión del uso público, y el filtraje de usuarios hacia otras áreas más resistentes.

\section{En el rango local:}

II.1. Los cerros del interior, que acogen ermitas y áreas de picnic que deben mantener un bajo nivel de uso local asegurando así su conservación como patrimonio natural y cultural.

II.2. AURIMN periurbanas, próximas a los focos de demanda recreativa, que atendiendan a las demandas de recreación intensiva, concentrando el uso y gestionadas con técnicas fuertemente intervencionistas como la recreación de hábitats con este objeto. 


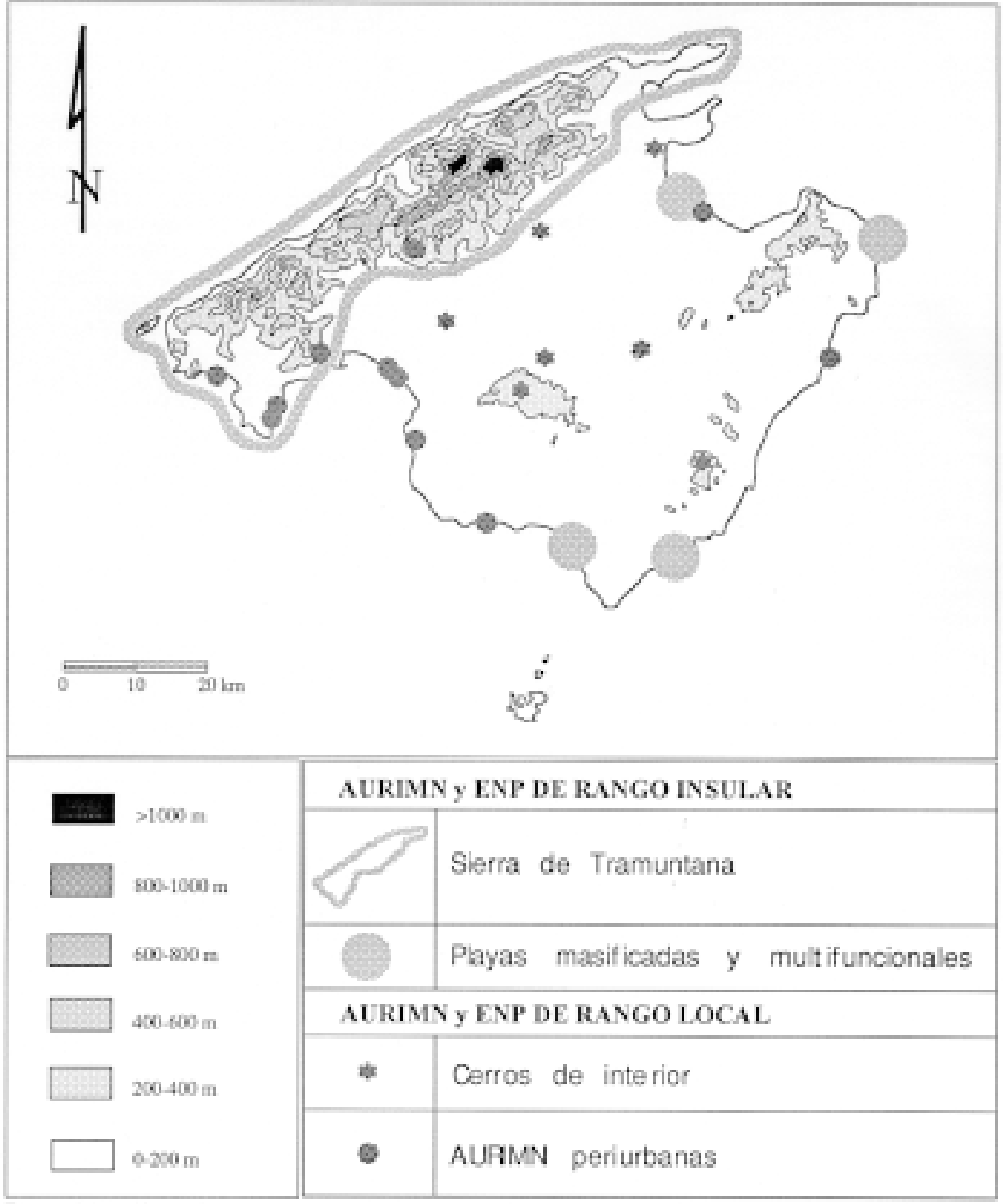

Fienser elabeenión poopia

MAPA 5. Propuesta de red jerarquizada y especializada de ENP y AURIMN de Mallorca

\section{Reflexión final a modo de conclusión}

El trabajo que se presenta se planteó bajo la óptica del interés por las perturbaciones que, en los ENP, provocan las actividades de ocio ${ }^{6}$. Pero su posterior desarrollo, junto con la reconducción que debemos agradecer a nuestro director, lo orientó a enfocar más y

$6 \quad$ Al hilo de nuestra tesis de licenciatura (BLÁZQUEZ, 1989). 
mejor las demandas humanas, en este caso la demanda recreativa real, que son el verdadero objeto de la Geografía. Éste es el significado de su subtítulo, «El alcance del ocio en el medio natural de Mallorca»: añadir la variable más geográfica, el uso antrópico, al campo de la planificación y gestión del medio natural.

Por supuesto que, más tratándose de un ejercicio pionero en esta materia en nuestro ámbito de estudio, será necesaria mucha más investigación al respecto, atendiendo sobre todo a la propia fuente de la demanda: las necesidades, preferencias y conveniencias de la población residente y turística de Mallorca.

\section{Referencias bibliográficas}

BAIRD, I.A. i IVE, J.R. (1989): «Using the LUPLAN Land Use Planning Package to Implement the Recreation Opportunity Spectrum Approach to Park Management Planning». In Journal of Environmental Management, $\mathrm{n}^{\circ} 29$, pp. 249-262.

BLÁZQUEZ, M. (1989): «Els impactes ambientals de les instal.lacions i activitats turístiques a les Illes Balears». Memoria de Licenciatura, Departament de Ciències de la Terra, Universitat de les Illes Balears. Inédito.

BLÁZQUEZ, M. (1992): «El Govern Balear i la Llei d’Espais Naturals. De la seva explotació publicitària al buidament del seu contingut», a El Mirall, n 51, pp. 14-15. Enero-febrero, 1992.

BLÁZQUEZ, M. (1993): «Mass tourism use of Majorcan natural sites. Proposals towards Sustainable Tourism Planning and Management. The cases of Sa Calobra, Sa Punta de N'Amer and Es Trenc». Disertación del master en Rural and Regional Resources Planning. Universidad de Aberdeen y Universidad Robert Gordon. Inédito.

BLÁZQUEZ, M. (1995): «La Platja d'es Trenc, un exemple d'Espai Natural Protegit sotmès a forta pressió recreativa informal, amb superació dels llindars de capacitat de càrrega ecològica $\mathrm{i}$ recreativa». XIII Jornades d'Estudis Històrics Locals. Institut d'Estudis Baleàrics. Diciembre, 1994.

BLÁZQUEZ, M. (en prensa): «Activitats recreatives i turístiques i els seus impactes ambientals». In GOB (en prensa): La Serra de Tramuntana: Natura i Cultura. Moll, Palma.

BURTON, R.C.J. (1975): «La cabida del campo para el recreo. Metodología y resultados de los estudios ecológicos y psicológicos en Cannock Chase, Staffordshire». Ministerio de Agricultura, Instituto Nacional para la Conservación de la Naturaleza, Monografías n ${ }^{\circ} 3$. Madrid.

CAMPILLO, X. (1992): «El conflicte entre lleure i protecció de la natura en espais naturals protegits a Baden-Württemberg (Alemanya): protecció versus promoció». Geographisches Institut Eberhard Karls Universität Tübingen, Baden-Württemberg (Alemanya). Inédito.

CAMPILLO, X. (1994): «El concepto de parque natural en Baden-Württemberg (Alemania): el caso del Parque Natural del Alto Danubio». In Documents d'Anàlisi Geogràfica, 24, 1994, pp. 75-92. Departament de Geografia, Universitat Autónoma de Barcelona.

CARCELLER, X. (1986): «Els parcs naturals com a factor de promoció sòcio-econòmica». In Revista econòmica de Banca Catalana, $\mathrm{n}^{\circ} 79$. Barcelona.

COLE, D.N. (1982): «Wilderness Campingsite Impacts: Effect of Amount of Use». Intermountain Forest and Range Experiment Station, Research Paper INT-284. USDA, Forest Service, Ogden.

COLE, D.N. (1985): «Recreational Trampling Effects on Six Habitat Types in Western Montana». Intermountain Research Station, General Technical Report INT-350. USDA, Forest Service, Ogden.

COLE, D.N. (1989): «Wilderness Campsite monitoring methods: a sourcebook». Intermountain Research Station, General Technical Report INT-259. USDA, Forest Service, Ogden.

COPOT (1987): «Proposta d'Actuació relativa a les Àrees Naturals». Govern Balear. Palma.

CORDELL, H.K. et alii (1990): «An Analysis of the Outdoor Recreation and Wilderness Situation in the United States: 1989-2040». USDA Forest Service, Athens.

CORDELL, H.K. et alii (1993): «Effects of Subdivision and Access Restrictions on Private Land Recreation Opportunities». USDA Forest Service, General Technical Report RM-231, Fort Collins. 
De FRUTOS, M. (1993): «Análisis de la oferta de Espacios Recreativos en la Comunidad de Madrid. Modelos predictivos de su utilización en un ámbito de planificación». Escuela Técnica Superior de Ingenieros de Montes, Universidad Politécnica de Madrid, inèdita.

De LUCIO, J.V. et alii (1992): «El estado del conocimiento del Parque Regional de la Cuenca Alta del Manzanares: bases ecológicas para la conservación».- Centro de Investigación de Espacios Naturales Protegidos Fernando González Bernáldez, Serie Documentos no 2. Madrid.

De LUCIO, J.V. i MÚGICA, M. (1994): «Landscape preferences and behaviour of visitors to Spanish national parks». In Landscape and Urban Planning, 29, 145-160.

DÍAZ SEGOVIA, A. (1991): «Los planes de ordenación de recursos naturales. El caso del Parque Natural de Urkiola». In Prácticas para la planificación de espacios naturales», colección técnica. ICONA. Madrid.

DIVERSOS AUTORES (1992a): «Espais naturals de les Illes Balears». IBATUR. Palma.

DIVERSOS AUTORES (1992b): «Espacios naturales protegidos de España. Guías Periplo». Incafo, ICONA. Madrid.

ENGLISH, D.B.K. et alii (1993): «Regional Demand and Supply Projections for Outdoor Recreation», General Technical Report RM-230. USDA Forest Service, Athens.

EQUIP TRAMUNTANA (1988): «Pla Territorial Parcial de la Serra de Tramuntana» (6 vols.). Universitat de les Illes Balears/Conselleria d'Obres Públiques i Ordenació del Territori del Govern Balear. Palma.

ESPACIOS NATURALES (1995): «Información básica de los Planes de Protección de las Áreas Naturales de Especial Interés». Govern Balear, COPOT, Palma.

EUROSITE (1994): «Management and zonning in Conservation Areas». Eurosite. Wimereux.

EUROSITE (1996): «Workshops on generating income from natural areas». Eurosite. Wimereux.

GLYPTIS, S. (1981a): «Room to relax in the countryside». In The Planner, 67 (4), pp. 120-122.

GLYPTIS, S. (1981b): «People at Play in the Countryside». In Geography, 66 (4), pp. 277-285.

GLYPTIS, S. (1991): «Countryside Recreation». Longman. Essex.

GÓMEZ-LIMÓN, J. (1992): «Alternativas de uso recreativo en el Parque Regional de la Cuenca Alta del Manzanares: las vías pecuarias». Centro de Investigación de Espacios Naturales Protegidos Fernando González Bernáldez, Serie Documentos nº 8. Soto del Real, Madrid.

GÓMEZ-LIMÓN, J. et alii (1993): «El impacto de las actividades recreativas al aire libre sobre los espacios naturales». In Quercus, nº 90, pp. 18-23.

GÓMEZ-LIMÓN, J. et alii (1994): «Áreas Recreativas en la Comunidad de Madrid. Afluencia de Visitantes y Actividades Desarrolladas». Centro de Investigación de Espacios Naturales Protegidos Fernando González Bernáldez, Serie Documentos nº 14. Soto del Real, Madrid.

GÓMEZ-LIMÓN, J. y de LUCIO, J.V. (1993): «Efectos del pisoteo sobre comunidades pratenses por acción de actividades recreativas en espacios naturales», Centro de Investigación de Espacios Naturales Protegidos Fernando González Bernáldez, Serie Documentos nº 12. Soto del Real, Madrid.

GÓMEZ-LIMÓN, F.J. y DE LUCIO, J.V. (1994): «Recreational Use Model in a Wilderness Area». In Journal of Environmental Management, $\mathrm{n}^{\circ} 40$, pp. 161-171.

HAMMITT, W.E. y COLE, D.N. (1987): «Wildland Recreation. Ecology and Management». John Wiley \& Sons, Nova York.

HEUKEMES, N. (dir.) (1993): «Loving them to death? Sustainable tourism in Europe's Nature and National Parks». Federación de Parques Naturales y Nacionales de Europa, Kliemo (Bélgica).

INESE (1985): «Àrees a Protegir a Balears». Govern Balear, Palma.

JANER MANILA, G. (dir.) (1992): «Calendari de Festes de les Illes Balears i Pitiüses». Alta Fulla y Fundació Serveis de Cultura Popular, Barcelona.

JUBENVILLE, A. y TWIGHT, B.W. (1993): «Outdoor Recreation Management. Theory and Application». Venture Publishing, State College.

KNUDSON, D.M. (1984): «Outdoor Recreation». Macmillan P.C. Nova York.

KUSS, F.R.; GRAEFE, A.R. y WASKE, J.J. (1990): «Visitor Impact Management». National Park and Conservation Association, Washington, D.C.

LANCE, A.; THAXTON, R. y WATSON, A. (1991): «Recent changes in footpath width in the Cairngorms». In Scottish Geographical Magazine, vol. 107, n² 2, pp 106-109. 
Ley 4/1989, de 27 de marzo, de Conservación de los Espacios Naturales y de la Flora y Fauna Silvestres, del Gobierno Español. Boletín Oficial del Estado, nº 74, 28-3-1989, pp. 8.262-8.269.

Ley 1/1991, de 30 de enero, de espacios naturales y de régimen urbanístico de las áreas de especial protección de las Islas Baleares. Boletín Oficial de la Comunidad Autónoma de las Islas Baleares, no 31, 09-03-1991, pp. 1882-1889.

MACEWEN, E. \& I. (1982): «National Parks, conservation or cosmetics». George Allen \& unwin, Londres.

MARÍN CABRERA, C. (1991): «Planificación del suelo y de espacios protegidos». In Prácticas para la planificación de espacios naturales. ICONA. Madrid.

MARTIN, B.S. y UYSAL, M. (1990): «An Examination of the Relationship Between Carrying Capacity and the Tourism Lifecycle: Management and Policy Implications». In Journal of Environmental Management, $\mathrm{n}^{\mathrm{o}}$ 31, pp. 327-333.

MILES, C.W.N. y SEABROOKE, W. (1977): «Recreational Land Management». E. \& F.N. Spon, Londres.

MÚGICA, M. (1994): «Modelos de demanda paisajística y uso recreativo de los espacios naturales». Centro de Investigación de Espacios Naturales Protegidos Fernando González Bernáldez, Serie Documentos $n^{\circ} 16$. Soto del Real, Madrid.

NATURE CONSERVANCY COUNCIL (1988): «Site management plans for nature conservation». NCC. Peterborough.

ROGGENBUCK, J.W., WILLIAMS, D.R. y WATSON, A.E. (1993): «Defining Acceptable Conditions in Wilderness». In Environmental Management, Vol. 17, n 2, pp. 187-197.

SCHOFIELD, P. (ed.) (1995): «EUROSITE European Guide for the Preparation of Management Plans for protected and managed natural and semi-natural areas». Eurosite, Wimereux.

STANKEY, G.H. (1980): «A comparision of carrying capacity perceptions among visitors to two wildernesses». Intermountain Forest and Range Experiment Station», Research Paper INT-242. USDA, Forest Service, Ogden.

WATSON, A. (1991): «Increase of people on Cairn Gorm plateau following easier access». In Scottish Geographical Magazine, vol. 107, n 2, pp. 99-105. 